\title{
Disposición a pagar por la restauración ambiental del río Lerma en la zona metropolitana de La Piedad, Michoacán
}

\author{
Willingness to pay for environmental \\ restoration of the Lerma river in the \\ metropolitan area of La Piedad, Michoacan
}

\author{
Dante Ariel Ayala-Ortiz* \\ Francisco Abarca-GuZmán**
}

\begin{abstract}
The metropolitan area of La Piedad, Michoacan-Santa Ana Pacueco, Guanajuato, shares a section of the Lerma river, whose conditions have severe environmental and public health problems. This research has three aims: first, identify the public perception of the local environmental damage of the Lerma river; second, estimate citizen willingness to pay (WTP) for its environmental restoration; and third, calculate a monthly payment for environmental improvements. We used the Contingent Valuation Method. The WTP for improvements in environmental quality was estimated at 12224424 MXN a year. Finally, we propose a monthly fee of 23.89 MXN per household.
\end{abstract}

Keywords: environmental remediation, urban development, environmental and economical valuation.

\section{Resumen}

La zona metropolitana La Piedad, Michoacán-Santa Ana Pacueco, Guanajuato comparte una sección del río Lerma cuyas condiciones propician fuertes problemas ambientales y de salud pública. En esta investigación se pretende: primero, identificar la percepción ciudadana sobre el deterioro ambiental local del río Lerma; segundo, estimar la disposición a pagar (DAP) por el saneamiento y restauración ambiental del mismo; y tercero, calcular una cuota mensual de contribución para las mejoras ambientales. Para ello se utilizó el Método de Valoración Contingente. La DAP por el saneamiento fue estimado en 12,224,424 pesos anuales y la cuota mensual doméstica propuesta es de 23.89 pesos.

Palabras clave: restauración ambiental, desarrollo urbano, valoración económico-ambiental.

\footnotetext{
* Universidad Michoacana de San Nicolás de Hidalgo. Correo-e: daao@fevaq.net

** Centro de Investigación y Docencia Económicas, A. c. Correo-e: fabaguz@gmail.com
} 


\section{Introducción}

Las continuas y diversas descargas industriales, urbanas, agrícolas y pecuarias que el río Lerma recibe desde su nacimiento y a su paso por cinco estados de la república lo han clasificado como uno de los ríos interiores de mayor contaminación en México; existen secciones y épocas del año en las cuales se sobrepasa con mucho los límites permisibles de concentración de contaminantes (López-Hernández et al., 2007). Este problema se ve agudizado por el actual patrón de crecimiento urbano que ha venido fortaleciendo el proceso de concentración económica y poblacional en las urbes, especialmente en las ciudades y núcleos metropolitanos (Navarro et al., 2004).

Desde hace algunas décadas, la zona metropolitana interestatal que comprende las ciudades de La Piedad de Cabadas, Michoacán y Santa Ana Pacueco, Guanajuato (ZM) se ha visto amenazada por los severos problemas ambientales y de salud pública derivados de la contaminación del río Lerma. Durante la mayor parte del siglo pasado la zona fue víctima de fuertes inundaciones derivadas del desborde de las aguas de este río.

En esta zona, el cauce natural del Lerma da lugar a una amplia curva (meandro) que envuelve a la localidad de Santa Ana Pacueco y es contorno de toda La Piedad. En su dinámica natural, la creciente del río propiciaba desbordamientos en la parte baja de la ciudad, razón por la que en los años ochenta fue construido un canal de alivio de aproximadamente $2.4 \mathrm{~km}$ que unió artificialmente los extremos del meandro natural, desde entonces, se controla el flujo de agua que entra al meandro y al canal de alivio a través de un sistema de compuertas. Sin embargo, con el paso del tiempo, este cambio en la dinámica hidráulica de los $12.5 \mathrm{~km}$ del meandro natural ha sido origen de múltiples problemas ambientales y de salud pública: aguas estancadas en estado de descomposición, segmentos casi secos y lechos contaminados que, a su vez, trajeron problemas de proliferación de fauna nociva, mal olor y fuertes afecciones a la salud.

Estudios realizados en 2009 por investigadores de la Universidad Michoacana ${ }^{1}$ muestran que un alto porcentaje de menores de edad tiene antecedentes de afecciones gastrointestinales y broncopulmonares, así como parasitosis diversas que incluyen a la solitaria (Taenia spp.) en su forma larvaria o cisticerco, así como otros helmintos y protozoos (Álvarez et al., 2011). Tales estudios reportan la presencia de huevos de 12 géneros de helmintos, incluyendo Ascaris sp. Taenia spp. y Toxocara sp. entre otros.

${ }^{1}$ Estudio paralelo al presente trabajo y que forma parte del mismo proyecto de investigación global en el área temática correspondiente a salud pública, subproyecto "Salud del humano, animales domésticos y silvestres". 
De hecho, las propias autoridades de salud en la entidad han reconocido que son aún más graves las consecuencias ocasionadas por el uso de pesticidas e insecticidas para combatir la fauna nociva, lo que repercute directamente en numerosos casos de leucemia infantil, registrados por el sector salud (Ruiz, 2009).

En este trabajo se presentan los resultados relativos a tres objetivos de una investigación más amplia. ${ }^{2}$ En primer lugar se indaga sobre la percepción de los habitantes de la zona relacionada con la situación de deterioro ambiental en la cual se encuentra el meandro del río Lerma que atraviesa la ZM. En segundo lugar se estima la disposición a pagar (DAP) que tiene la ciudadanía para contribuir con el saneamiento y restauración ambiental del meandro. Por último, se calcula una cuota mensual que potencialmente podría aplicarse con la finalidad de asegurar la efectiva contribución ciudadana a las mejoras ambientales. Este trabajo se justifica en la medida en la que contribuya a la generación de alternativas de restauración y manejo de la degradación ambiental, incluyendo la participación de la ciudadanía.

\section{La valoración contingente en el estudio de los problemas económico ambientales}

El Método de Valoración Contingente (MVC) permite estimar la máxima DAP de un individuo por la provisión o mejora de un bien de no mercado, para ello se recurre a la generación de mercados hipotéticos (MogasAmorós, 2004) mediante los cuales los potenciales usuarios de bienes o servicios ambientales le asignan un valor derivado de los beneficios que éstos pueden proporcionarles (Azqueta et al., 2007). La principal ventaja que se le reconoce al Mvc es que puede cuantificar valores de no-uso (Azqueta, 1994; Sarmiento y Prieto-Rodríguez, 2005).

Con relación a la calidad del agua, la valoración económico ambiental ha sido aplicada con fines diversos que van desde la mera conservación hasta la sustentabilidad de servicios ambientales proporcionados por recursos hídricos. En tales trabajos se destaca la importancia de la valoración económica ambiental para la toma de decisiones relacionada con el cuidado y la calidad del agua (Arrow et al., 1993).

\footnotetext{
${ }^{2}$ Investigación realizada bajo el auspicio de Fomix-Conacyt, proyecto no. 73881, "Saneamiento del cauce natural (meandro) del río Lerma e integración del mismo a la dinámica urbana de La Piedad, Michoacán”; proyecto multidisciplinario que contó con la participación de 42 investigadores de la Universidad Michoacana de San Nicolás de Hidalgo, entre ellos los autores del presente, realizado con la finalidad de generar proyectos y alternativas viables para la resolución de la problemática ambiental y urbana generada por la degradación del meandro del río Lerma en la zona metropolitana de La Piedad-Santa Ana Pacueco.
} 
Por lo general, los individuos son sensibles ante una serie de aspectos relacionados con la calidad del agua y, por lo tanto, es posible fundamentar un estudio de valoración contingente mediante el cálculo de la disposición a pagar por mejoras en esos aspectos, Egan et al. (2009) desarrollaron un modelo logit mixto sobre el uso recreativo de un lago; encontraron que las estimaciones de DAP son determinadas por las características socioeconómicas de los hogares.

Lo anterior también se ha visto reflejado en estudios realizados en nuestro país como el de Soto-Montes de Oca (2007), quien a partir del valor económico del agua para uso doméstico redefine las políticas de precios del agua potable, generando información básica para mejorar el abasto de este bien en la ciudad de México. Al seguir a esta autora, la DAP por parte de los hogares es básica en la solución de problemas ambientales de zonas urbanas, siempre que dicha disposición sea una manifestación de la capacidad de pago de la población, así como de sus preferencias por niveles específicos de calidad ambiental.

Con la información otorgada por los usuarios directos es posible diseñar políticas que compatibilicen, con mayor facilidad, objetivos de eficiencia económica, equidad social y uso racional de un recurso escaso. Sobre esta temática existen múltiples antecedentes, por ejemplo, en Colombia se realizó un estudio para determinar la viabilidad financiera de un proyecto para el tratamiento de aguas residuales en una localidad rural aplicando el MVC, se pretendía conocer si el nivel de recuperación de costos permitiría cubrir cuando menos los costos de operación y mantenimiento de una planta de tratamiento (Rojas-Padilla et al., 2001). El estudio determinó que la DAP promedio de los habitantes de la localidad ascendía a 0.57 dólares mensuales por habitante, se estimó así la sostenibilidad financiera del proyecto por etapas.

En otro estudio realizado en dos ciudades de la costa del Pacífico de Costa Rica, hecho con el objetivo de determinar la DAP por mejoras en la calidad del agua costera, se asignó un valor de manera directa a las mejoras ambientales derivadas de los proyectos de saneamiento, estimando los beneficios potenciales de éstos antes de su implementación (Barton, 2007).

De igual modo, Bernstein et al. (2013) al usar Mvc estiman que los hogares en el noreste de los Estados Unidos tienen una DAP de us 12 dólares mensuales para financiar proyectos dirigidos a mejorar el acceso a las amenidades del río Susquehanna, y de us 10.46 por eliminar los riesgos derivados de la fractura de rocas subterráneas por la extracción de gas natural.

En México, Ojeda et al. (2008) utilizaron el Mvc para estimar el valor económico de los servicios ambientales (sA) derivados de la restauración ecológica en afluentes del río Yaqui, Sonora, encontraron que los hogares 
pagarían un promedio de 73 pesos mensuales por la recuperación de diversos SA, y concluyeron que la DAP se relaciona con el ingreso, nivel educativo, número de niños en el hogar, entre otros.

Con otro estudio Ibarrarán et al. (2003) identificaron los determinantes de la DAP por mejoras en la calidad ambiental derivadas de la construcción de un relleno sanitario de residuos sólidos en el centro-oeste del estado de Puebla, encontraron que la DAP está determinada por el nivel de ingreso, la edad y la confianza en las autoridades gubernamentales.

\section{Metodología}

\subsection{Descripción del sitio de estudio}

La Piedad de Cabadas, Michoacán, y Santa Ana Pacueco en Pénjamo, Guanajuato, son localidades colindantes separadas de manera natural por el lecho del río Lerma (figura I). Recientemente, estas dos localidades formaron la zona metropolitana La Piedad-Santa Ana Pacueco para promover un desarrollo conjunto y atender problemas comunes.

Ambientalmente, el problema más grave que enfrenta esta zM es el creciente deterioro ecológico del río Lerma. Tal situación ha traído, desde hace años, molestias y complicaciones para la mayoría de los habitantes quienes, a su vez, resultan en buena medida responsables de la contaminación. Si bien la contaminación del Lerma es resultado de descargas urbanas, agropecuarias e industriales que recoge a su paso (López-Hernández et al., 2007), existen estudios que destacan la propia influencia de las descargas de esta ZM en la contaminación del río. ${ }^{3}$

\subsection{El Método de Valoración Contingente (MVC)}

Mediante el MvC calculamos la cantidad promedio que los hogares de esta ZM estarían dispuestos a pagar por revertir el deterioro ambiental del meandro. Para ello, a los entrevistados se les planteó un escenario hipotético sobre la mejora de la calidad del cauce natural del río y su ribera con un proyecto integral de saneamiento y restauración que contemplase desde una mejor administración del agua que entra al cauce natural del río, hasta la construcción de áreas jardinadas, parques recreativos, pasos peatonales y vehiculares. Una vez embozado el escenario hipotético se les interrogó sobre su DAP para mejorar la situación ambiental del meandro y su zona de influencia.

\footnotetext{
${ }^{3}$ De acuerdo con Sotelo et al. (2005), la zona metropolitana de La Piedad es el principal generador de aguas residuales de la Subcuenca Lerma conformada por 18 municipios de los estados de Guanajuato, Jalisco y Michoacán, entre ellos el de La Piedad.
} 


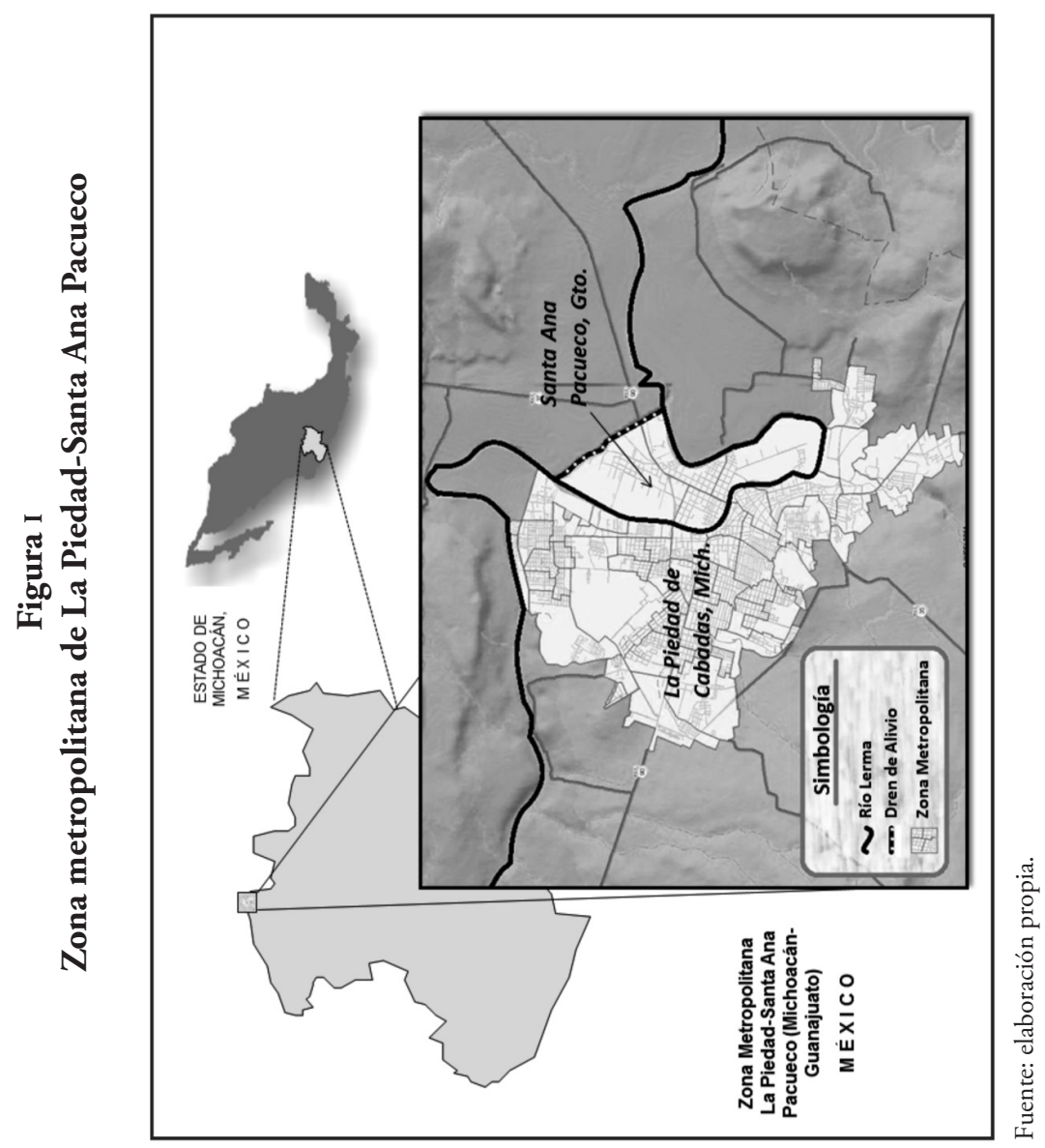




\subsection{El instrumento de aplicación}

Las entrevistas se realizaron a una muestra representativa de hogares de La Piedad de Cabadas y Santa Ana Pacueco, se aplicó un cuestionario para indagar sobre aspectos como i) la problemática general de la localidad, ii) la valoración económico ambiental, iii) la cultura del agua, iv) el servicio público de agua potable, y v) el perfil socioeconómico del entrevistado. ${ }^{4}$ El cuestionario final fue previamente depurado con una prueba piloto. La sección de interés para el estudio corresponde a la valoración económico ambiental del río.

Se comenzó por hacer un resumen de la situación actual del meandro del Lerma, describiendo cuál podría ser el escenario futuro sin acciones. En contraposición a este escenario se planteó la alternativa de implementar un proyecto para revertir el deterioro del cauce natural del río, incrementando la cantidad y calidad del agua. Ante el escenario futuro con acciones el entrevistado debió decidir si estaría dispuesto a pagar.

Para esto, tal como sugieren Labandeira et al. (2007), se planteó una pregunta de formato binario con el fin de conocer si el individuo estaría dispuesto, o no, a pagar por las mejoras descritas. Si estaba a favor se le cuestionaba el monto en pesos que estaría dispuesto a pagar mensualmente, a través del cobro realizado por el organismo local encargado de operar los servicios de alcantarillado y agua potable, ${ }^{5}$ como lo sugiere Arrow et al. (1993).

Siguiendo a Riera et al. (2005), la pregunta sobre el monto de DAP por cada hogar se realizó en formato abierto, esto es, a partir de una cantidad inicial de cinco pesos/mes, el entrevistado expresa una cantidad que para él resulta adecuada, dados su nivel de ingreso, su situación de egresos, y el beneficio que espera recibir a cambio.

Para el monto máximo de DAP se formuló una segunda pregunta de tipo subasta, se ofreció como incentivo la certeza de que el proyecto se realizaría con la participación obligatoria de toda la población y sin desvío de recursos, ${ }^{6}$ tras lo cual se preguntó la DAP máxima (se redujeron sesgos de conducta individual por falta de información).

${ }^{4}$ Los aspectos iii) y iv) forman parte de otro reporte de investigación.

${ }^{5}$ Como ha comprobado Ivehammar (2009), los participantes en este tipo de estudios muestran una mayor disposición a aceptar el pago cuando el vehículo de pago está ya establecido - tal como el cobro de servicios o ciertos impuestos- que cuando se propone una donación voluntaria (lo que lleva aparejado el comportamiento estratégico del free rider).

${ }^{6}$ Cuando se plantea el pago como algo obligatorio los participantes deducen que la elusión es algo casi imposible por lo que la disposición a pagar incrementa. Este comportamiento es similar al observado cuando el pago se plantea como algo que se aplicará a la población en su conjunto y no sólo a los usuarios directos del bien (Ivehammar, 2009). 
En adición, al considerar que la construcción de un mercado para los bienes ambientales puede presentar ciertos problemas que se traducen en fuentes de error o sesgo en las estimaciones obtenidas, se aplicaron los mecanismos sugeridos por la literatura para evitar los sesgos ocasionados por (i) comportamiento estratégico, (ii) comportamiento hipotético, (iii) efecto de inclusión, (iv) efecto de la información, y (v) efecto de anclaje. ${ }^{7}$

\subsection{Diseño muestral}

El muestreo estadístico fue de tipo aleatorio estratificado, se tomaron como marco muestral los padrones de usuarios domésticos del Sistema de Agua Potable, Alcantarillado y Saneamiento de La Piedad (sapas) y del Comité de Agua Potable de Santa Ana Pacueco (CAPAs). En total, se aplicaron 875 encuestas (611 en La Piedad y 264 en Santa Ana Pacueco), que aseguran $95 \%$ de confianza con un error máximo del 3\% para la muestra conjunta. La estratificación fue establecida según tipo de usuario doméstico (cuadro 1).

El tipo de entrevista fue individual y mediante visita domiciliar, se hizo el levantamiento de la información entre los meses de agosto y septiembre de 2009, con una aplicación piloto realizada en julio del mismo año.

\subsection{Estimación del Modelo de Regresión Logística}

El MvC se sustenta en la llamada teoría de la utilidad aleatoria, la cual sostiene que la utilidad que un consumidor obtiene puede ser estimada mediante los componentes aleatorios o contingentes que manifiestan los individuos. ${ }^{8}$ Para conocer cuáles son las variables que determinan la aceptación o rechazo del pago por mejoras ambientales en cada estrato realizamos varios análisis de correlación y regresiones logarítmicas

\footnotetext{
${ }^{7}$ Para una buena revisión de la naturaleza de estos sesgos y la forma de superarlos o minimizarlos se puede recurrir a Labandeira et al. (2007:154-158), así como a Harrison y Rutström (2008).

${ }^{8}$ Para el cálculo de la DAP de una población se utiliza información de las preferencias individuales. Si bien se puede observar cierta información, existen otros componentes desconocidos los cuales llevan a que la función de utilidad se trate como una función con un componente aleatorio, que debe ser tratada en términos de probabilidad. Es decir, si atendemos solamente al nivel de precios, al nivel de ingresos, al cambio en el bien ambiental y al pago propuesto, no es posible afirmar que un individuo aceptará el cambio, sin embargo, es posible calcular la probabilidad de que lo haga. Así, la probabilidad de que el cambio propuesto sea aceptado es el resultado de una ponderación de probabilidades: si el nivel de utilidad sin la mejora ambiental es menor a la utilidad obtenida con esa mejora, entonces es posible que un individuo esté a favor de un cambio (Riera et al., 2005). Por esta característica es necesario recurrir a un modelo de regresión logística binaria o logit (modelos de regresión de respuesta cualitativa), cuya principal peculiaridad es que la variable de respuesta puede ser de naturaleza cualitativa, y puede presentar, o no, cierto atributo (Gujarati, 2003). De hecho, el objetivo de estos modelos es encontrar la probabilidad de que un evento suceda o no.
} 


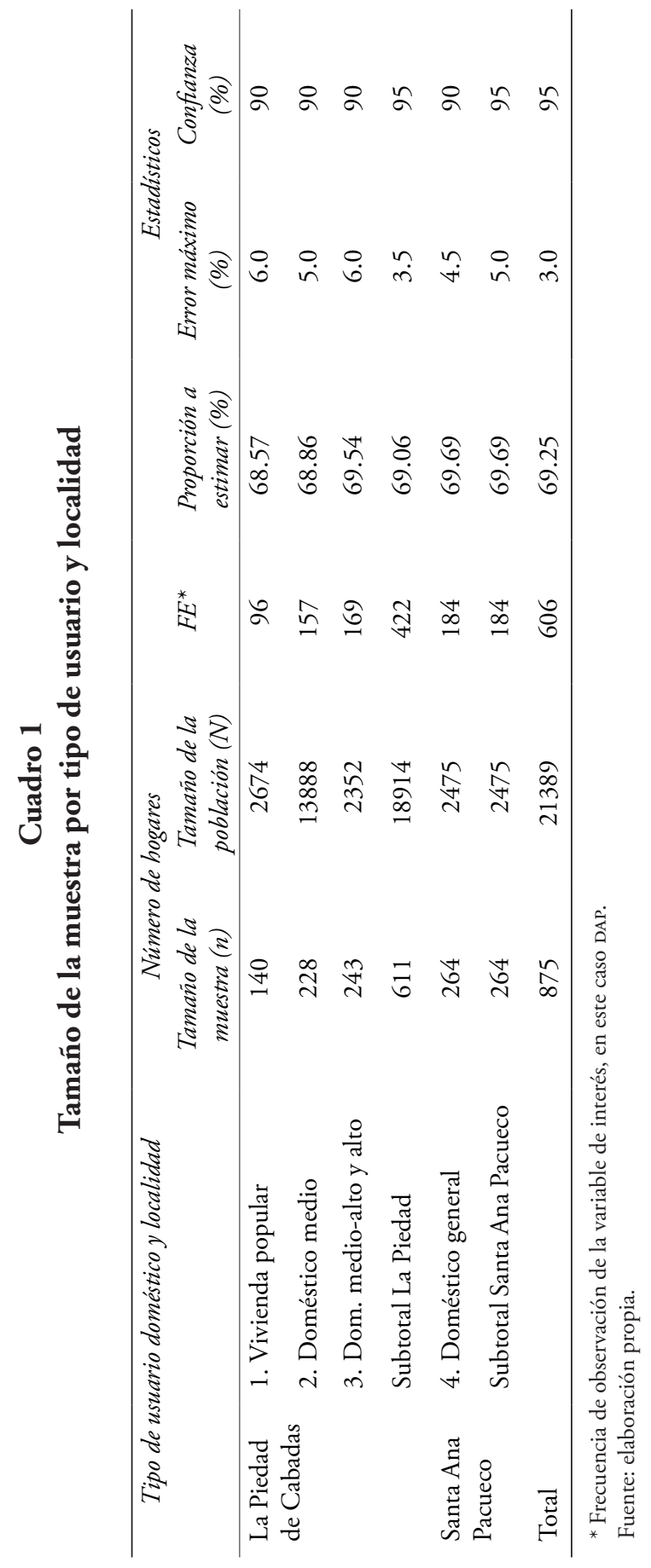


sugeridos para este tipo de estudios (Pucutay-Vásquez, 2002; Riera et al. 2005; Labandeira et al., 2007). De esta manera, obtuvimos seis variables que explican la probabilidad de DAP.

En el cuadro 2 se describe cada variable, su clasificación, simbología utilizada y el sentido de la relación esperada de cada variable explicativa con la variable dependiente. Por ejemplo, esperamos que la edad del entrevistado tenga una relación inversa con su DAP ya que, mientras mayor sea ésta, menor será la probabilidad de que acepte realizar el pago. Ejemplo contrario, el ingreso, variable que a medida que incrementa, se espera que mayor sea la probabilidad de aceptar el pago (Franzen y Vogl, 2013).

Es importante señalar que no todas las variables seleccionadas determinan la probabilidad de DAP en todos los estratos. Por ejemplo, en el estrato doméstico medio la variable cultura del agua no influye en la DAP, pero sí lo hace en el doméstico bajo.

\section{Resultados}

\subsection{Identificación de la problemática}

De acuerdo con los resultados obtenidos a partir del estudio a hogares, para los habitantes de esta zM la contaminación del río es uno de los principales problemas que enfrentan, es superado solamente por la inseguridad, pero considerada más grave aún que el desempleo, la falta de limpieza en las calles, la falta de pavimentación e incluso que la marginación o la pobreza (cuadro 3). Obsérvese el comportamiento similar y proporcional en las respuestas de ambas localidades.

Para los habitantes de la ZM, la contaminación del río Lerma es ocasionada en buena medida por la acción misma de la población, ya que a su cauce se arrojan toda clase de desechos; lo anterior concuerda con Ruiz (2009) quien señala que el meandro se ha convertido en una suerte de drenaje a cielo abierto, en gran parte generado por las descargas locales (Sotelo et al., 2005). De este modo, se halló que más de 60\% de los hogares encuestados consideró que el principal afecto negativo del deterioro ambiental del río es la fauna nociva, por la presencia de mosquitos, moscas, cucarachas y roedores.

Alrededor de $20 \%$ de la población encuestada aseguró haber padecido alguna enfermedad atribuible a la condición degradada del Lerma, con una mayor incidencia para los estratos de doméstico bajo y doméstico medio, a diferencia del doméstico alto y medio alto. A partir de la submuestra anterior $(n=168)$, para el caso de La Piedad, las enfermedades asociadas al deterioro ambiental del Lerma reportadas con mayor frecuencia fueron infecciones en la piel, infecciones en las vías respiratorias, fiebre tifoidea, 


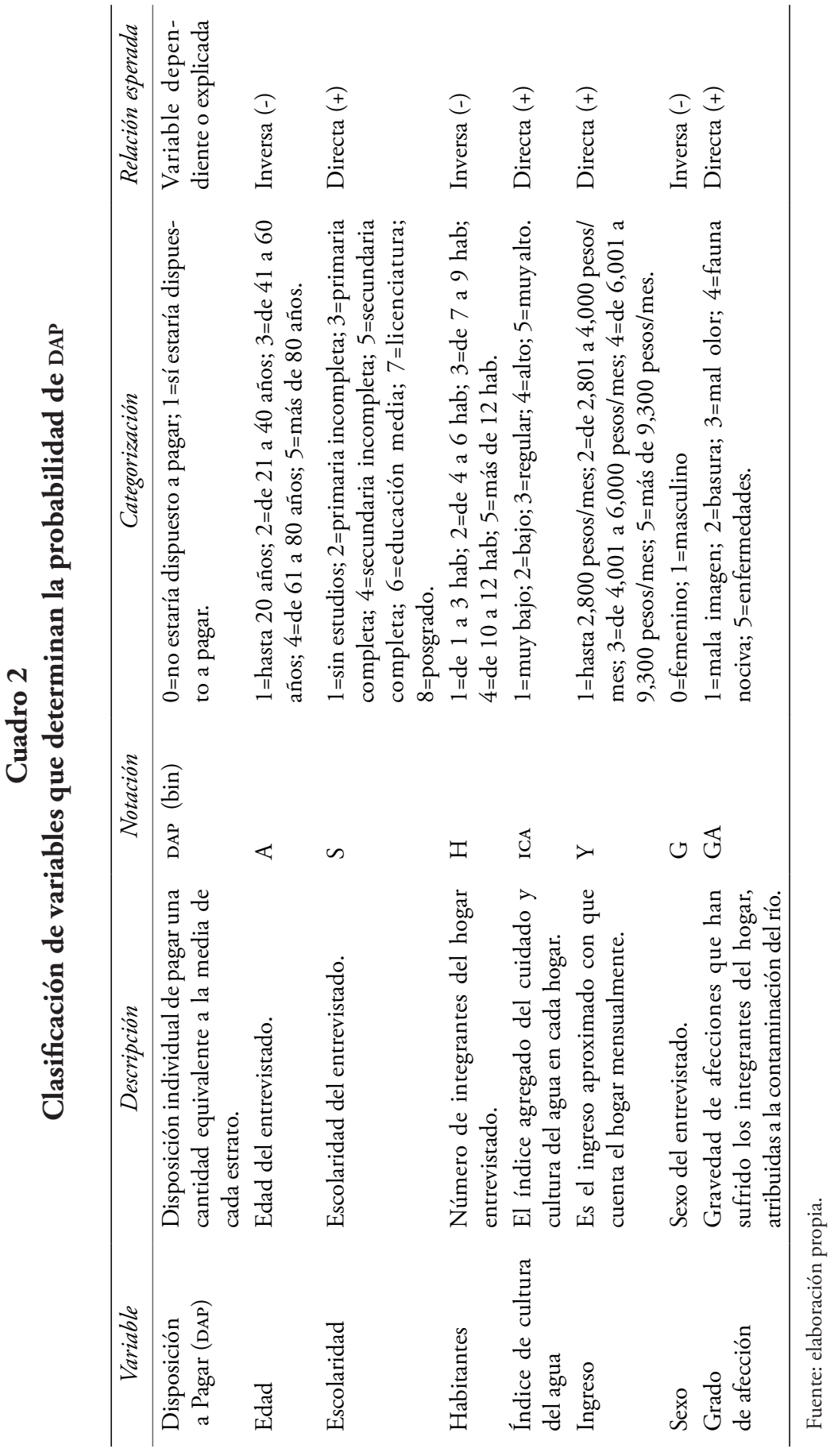




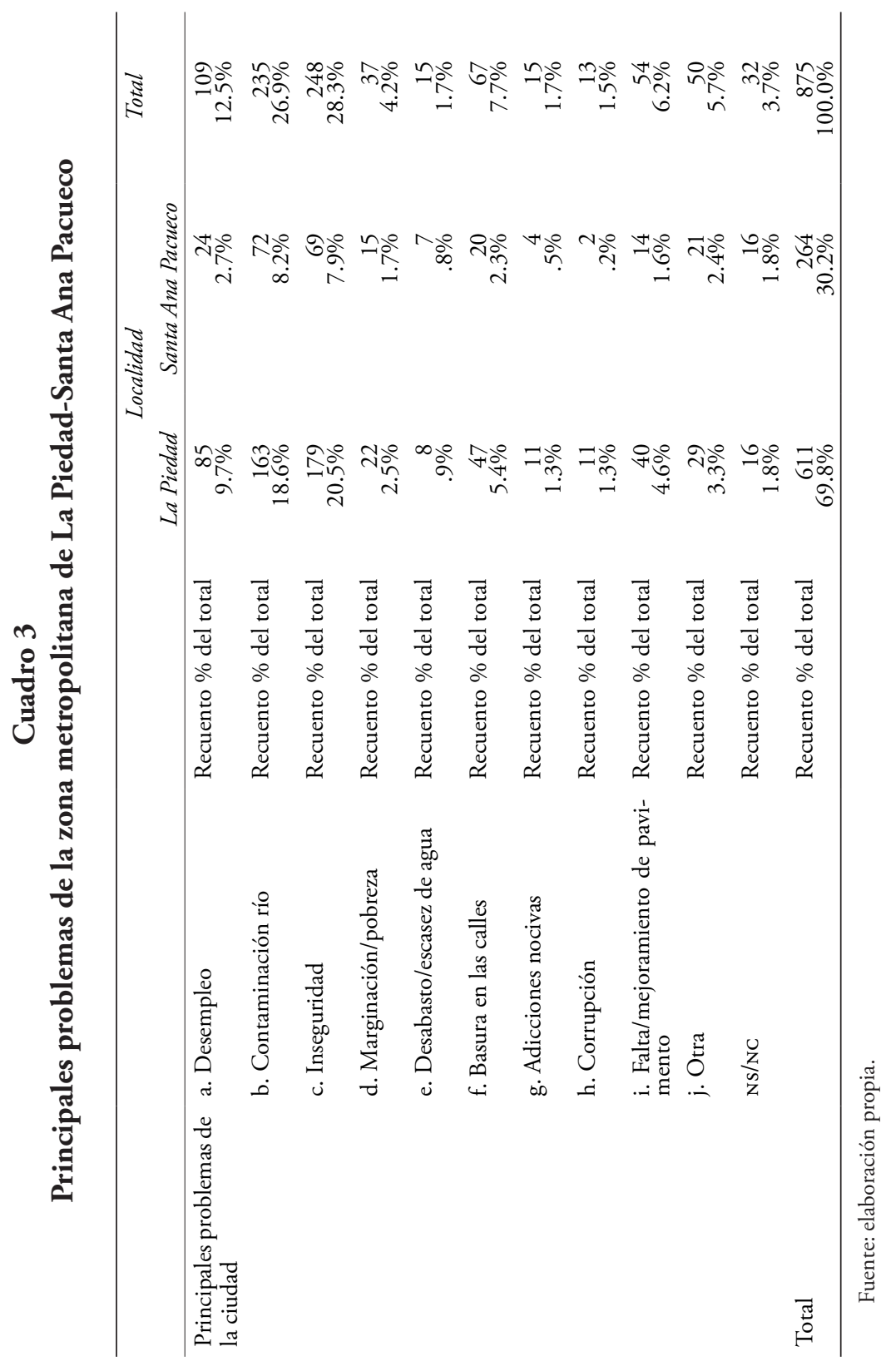


amebiasis, gastroenteritis y cáncer; en el caso de Santa Ana Pacueco fueron infecciones en la piel seguidas de disentería, infecciones en vías respiratorias y fiebre tifoidea.

En ambas localidades, más de $85 \%$ de los entrevistados aseguró que en sus hogares se hace uso de insecticidas. Esto coincide con lo señalado por las autoridades de salud en la entidad, quienes aseguran que el uso de pesticidas e insecticidas para combatir la fauna nociva han tenido incidencia directa en el incremento de los casos de leucemia infantil, enfermedades broncopulmonares y alergias registrados en la zona (Ruiz, 2009). Aproximadamente, uno de cada cinco hogares que utilizan insecticidas como medida de control de fauna nociva (submuestra $n=148$ ) han presentado problemas de salud, las más frecuentes son las relacionadas con el sistema respiratorio (50\%). Las alergias en ojos o piel corresponden a $20 \%$ de los casos afectados por el uso de insecticidas.

\subsection{Participación ciudadana en las mejoras ambientales}

Una vez que se dio a conocer a los entrevistados la situación de deterioro ambiental del meandro del Lerma se les comentó que esta situación podría ser reversible tomando las medidas necesarias. Así, al plantear un escenario hipotético donde la ciudadanía fuera llamada a votar a favor o en contra de una aportación mensual para contribuir al saneamiento del Lerma se obtuvo que $81.9 \%$ (717/875) de los entrevistados de la zM estarían a favor del pago y, sólo $17.1 \%$ rechazaría la propuesta (cuadro 4).

A la submuestra que se declaró a favor del pago $(n=717)$ también se le preguntó acerca del monto que podría aportar de manera mensual sin afectar su situación económica. Los montos obtenidos van desde los cinco hasta los 150 pesos mensuales por hogar. Las cantidades que se mencionaron con mayor frecuencia estuvieron ubicadas, en primer lugar, por debajo de los 10 pesos mensuales (25\% de los casos), en segundo lugar, entre los 11 y 20 pesos mensuales (18\% de los casos en La Piedad y $22 \%$ en Santa Ana), y en tercer lugar se ubican montos mayores que van de los 40 a los 50 pesos con 17\% de los casos en La Piedad y 19\% en Santa Ana (figura II).

Para conocer los motivos por los cuales la otra submuestra $(n=150)$ votó en contra del pago, se pusieron a su consideración varias razones. Una de ellas aludía a la carencia de condiciones económicas con la cual coincidieron casi 40\% de los casos en La Piedad, y 70\% en Santa Ana. Otro argumento importante fue la desconfianza de la ciudadanía hacia las autoridades (más de 60\% de la submuestra). 


\section{Figura II}

Monto mensual de la DAP máxima por hogar, según estrato y localidad (submuestra a favor del pago $n=717$ )

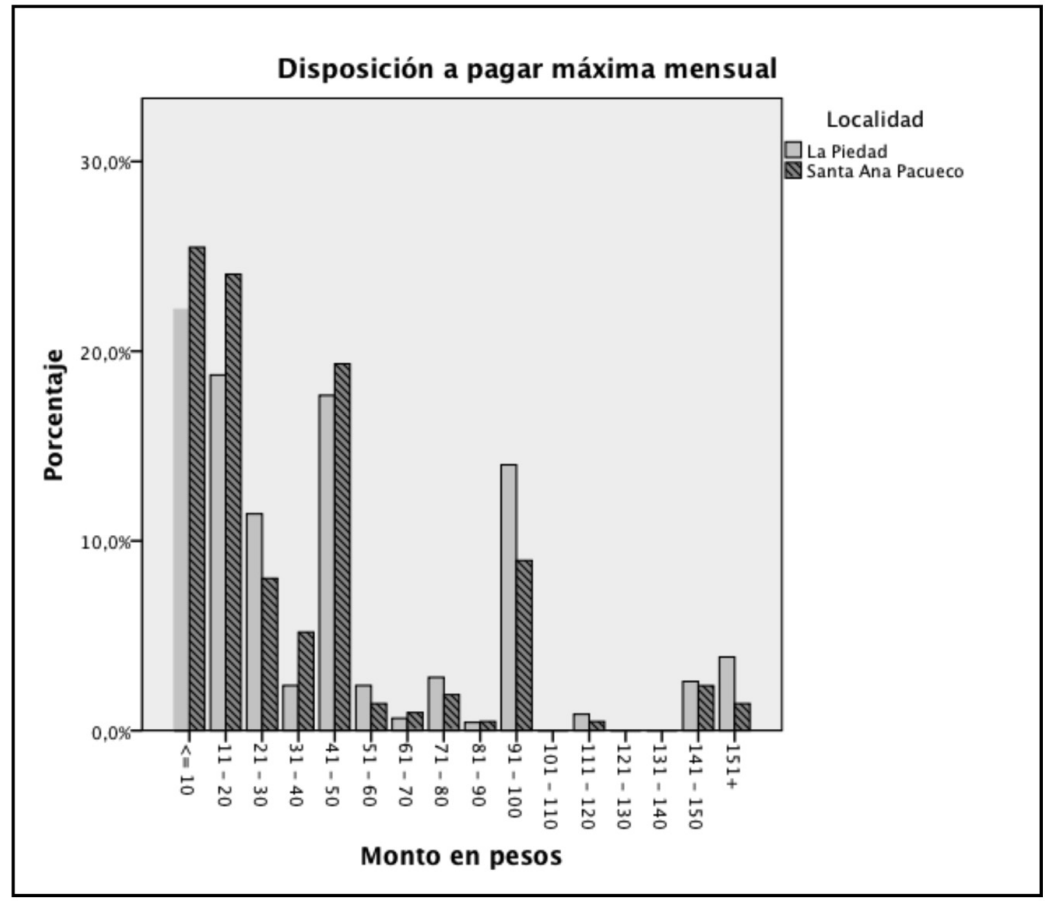

Fuente: elaboración propia.

\subsection{Probabilidad de contribuir con el pago en la Zona Metropolitana}

El estudio de valoración contingente en la localidad michoacana se aplicó a un total de 611 hogares, equivalentes a $69.8 \%$ de la muestra recogida. A continuación se presentan los resultados del modelo logit estimado para cada estrato de la ZM.

\subsubsection{Estrato doméstico bajo, La Piedad}

En total se entrevistaron 140 hogares pertenecientes al estrato doméstico bajo, equivalente a $22.91 \%$ de la submuestra de La Piedad. Los resultados del modelo de regresión logarítmica, calculado a partir de estos datos, se resumen en el cuadro 5. 


\section{Cuadro 5}

Modelo de regresión para el estrato doméstico bajo, La Piedad

\begin{tabular}{|c|c|c|c|}
\hline \multicolumn{4}{|c|}{$\begin{array}{l}\text { Estrato: } D B \\
\text { Resultados de la regresión } \\
\text { Variable dependiente: DAP (bin) } \\
\text { Método: logistico binario }\end{array}$} \\
\hline Variable & B & Sig. & $e^{\mathrm{B}}$ \\
\hline $\mathrm{Y}$ & .407 & .038 & 1.502 \\
\hline$S$ & .395 & .082 & 1.484 \\
\hline G & -.862 & .122 & .422 \\
\hline ICA & .504 & .147 & 1.655 \\
\hline Constante & -5.007 & .006 & .007 \\
\hline & & & R2 de Cox y Snell=0.121 \\
\hline
\end{tabular}

Fuente: elaboración propia.

A partir de los resultados mostrados en ese cuadro, podemos construir el modelo $Z i$ del que obtendremos el valor logit que permitirá calcular la probabilidad de que un hogar acepte el pago propuesto para mejoras ambientales.

Cada coeficiente (B) es dependiente parcial que mide el cambio en el logit estimado, correspondiente a una unidad de cambio del valor de la regresada (variable dependiente) si las demás variables explicativas permanecen constantes 9 (Aguayo, 2007). De esta manera, el modelo que estima la probabilidad de que un hogar del estrato doméstico bajo tenga una DAP favorable por las mejoras ambientales es:

$$
Z_{\mathrm{DB}}=-5.007+0.407(\mathrm{Y})+0.395(\mathrm{~S})-0.862(\mathrm{G})+0.504(\mathrm{ICA})
$$

Una interpretación más significativa de los resultados obtenidos es la expresada en términos de probabilidades al obtener el antilogaritmo de los coeficientes de la pendiente (Gujarati, 2003). De esta manera, si tomamos el antilogaritmo del coeficiente $\mathrm{Y}$, obtenemos que $\mathrm{e}^{0.407} \approx 1.5$. Este resultado sugiere que un hogar al que ingresan hasta 4,000 pesos mensuales (categoría 2 de ingreso) tiene 1.5 veces más probabilidades de

${ }^{9}$ Por lo tanto, el coeficiente de Y igual a 0.407 implica que, siempre que las demás variables no cambien, si Y incrementa en una unidad, entonces en promedio el logit estimado aumentará casi 0.4 unidades, lo que corrobora que la relación existente entre la variable Y, y la variable dependiente, es directa (+) tal como se supuso anteriormente. De hecho, las demás variables explicativas incluidas en este caso guardan también el tipo de relación supuesto en el cuadro 5. Así, de incrementar en una unidad las variables escolaridad (S) y el indice de cultura del agua (ICA), el logit estimado incrementará en 0.395 y 0.504 , respectivamente, las demás variables permanecerán constantes. Por el contrario, de incrementarse en una unidad la variable sexo $(\mathrm{G})$, y al permanecer las demás variables sin cambios, el valor del logit disminuirá en 0.395 . 
DAP que un hogar que ingresa menos de 2,800 pesos al mes (categoría 1 de ingreso).

Supongamos entonces que, a partir de los datos contenidos en el cuadro 6, deseamos conocer la probabilidad de que el hogar 1 acepte dar el pago por mejoras ambientales. Al sustituir los datos reales de este hogar en el modelo estimado, observaremos que el logit obtenido en este caso es -1.2908 , por lo que la probabilidad estimada de aceptar el pago es 0.2157 . Esto es, un hogar con las mismas características de este primer caso muy seguramente rechazaría el pago propuesto. ${ }^{10}$ Por el contrario, un hogar como el 11 del cuadro 6, seguramente aceptaría el pago propuesto ya que su probabilidad de hacerlo es de 0.58 (casi 60\%), lo que resulta tanto de un mayor nivel de ingreso $(\mathrm{Y})$ como de una cultura del agua (ICA) más elevada respecto al primer caso analizado. El comportamiento positivo de esta última variable respecto a la DAP es consistente con lo reportado por Husted et al. (2013), quienes señalan que una actitud o cultura más pro-ambiental aumenta la DAP marginal; en nuestro caso, DAP máxima.

\section{Cuadro 6 \\ Ejemplo de la probabilidad de la DAP para contribuir con las mejoras ambientales para dos hogares del estrato doméstico bajo, con distintas características}

\begin{tabular}{ccccccc}
\hline & & & \multicolumn{5}{c}{ Variables } \\
\hline & Hogar & Estrato & $S$ & $Y$ & $G$ & ICA \\
& 1 & DB (1) & 2 & 1 & 0 & 5 \\
& $\cdot$ & $\cdot$ & $\cdot$ & $\cdot$ & $\cdot$ & $\cdot$ \\
& $\cdot$ & $\cdot$ & $\cdot$ & $\cdot$ & $\cdot$ & $\cdot$ \\
\hline
\end{tabular}

Fuente: elaboración propia.

La bondad de ajuste alcanzada por las variables del modelo estimado puede observarse a partir de la $\mathrm{R}^{2}$ de Cox y Snell ${ }^{11}$ equivalente en este caso a 0.121 (cuadro 5). Si bien este valor resulta bajo, se resalta que en los modelos con regresada binaria, la bondad de ajuste tiene una importancia secundaria, lo que interesa son los signos esperados de los coeficientes de la regresión para sus implicaciones prácticas ${ }^{12}$ (Gujarati 2003).

\footnotetext{
${ }^{10}$ Seguimos el criterio siguiente: el pago será rechazado cuando $\operatorname{Pr}<0.5$.

${ }^{11} \mathrm{La} \mathrm{R}^{2}$ de Cox y Snell es un coeficiente de determinación utilizado para estimar la proporción de varianza de la variable dependiente explicada por las variables explicativas.

${ }^{12}$ Para obtener detalles más específicos sobre este aspecto, el lector puede consultar el capítulo 15 de Gujarati (2003) donde se hace el análisis de esta cuestión.
} 
En resumen, en el estrato correspondiente a doméstico bajo, la probabilidad de que un hogar tenga una DAP positiva para revertir el deterioro del meandro del Lerma dependerá del nivel de ingresos mensual del hogar, de la escolaridad y sexo del jefe del hogar, así como de la cultura del agua presente. Incrementos en estos factores darán como resultado una mayor probabilidad de aceptar el pago propuesto, con excepción del sexo cuya relación inversa con el logit, indica que las probabilidades DAP serán menores cuando la decisión de hacerlo recaiga en un hombre.

\subsubsection{Estrato doméstico medio, La Piedad ${ }^{13}$}

El estudio de valoración contingente se realizó con 228 hogares pertenecientes al estrato doméstico medio (DM). A partir de la información obtenida de esta muestra, equivalente a $37.3 \%$ de hogares entrevistados en La Piedad, se estimó el logit que permite conocer la probabilidad de que la población de este estrato contribuya con una aportación mensual para el saneamiento del meandro del Lerma. De esta manera, el logit para estimar la probabilidad de que un hogar de este estrato acepte el pago propuesto es:

$$
Z_{\mathrm{DM}}=-0.707+0.374(\mathrm{~S})+0.527(\mathrm{G})-0.363(\mathrm{H})-0.225(\mathrm{~A})
$$

En este caso, el logit estimado reaccionará de manera positiva ante cambios unitarios del nivel de escolaridad $(\mathrm{S})$ y sexo $(\mathrm{G})$ del entrevistado. Sin embargo, disminuirá a medida que el número de habitantes del hogar (H), y la edad (A) del entrevistado sean mayores.

\subsubsection{Estrato doméstico medio-alto y alto, La Piedad}

En este último estrato de La Piedad se entrevistaron 243 hogares que equivalen a $39.8 \%$ de la muestra de la localidad. Así, se obtuvo que la probabilidad es determinada para este conjunto de hogares por variables como el indice de cultura del agua (ICA) y el nivel de ingreso del hogar (Y), así como por la edad (A) y sexo del entrevistado (G). Se encontró una relación inversa (negativa) de las dos primeras variables con la regresada, y una relación directa (positiva) entre ésta y las dos últimas variables.

\footnotetext{
${ }^{13}$ Para fines de exposición, en éste y los siguientes subapartados en los que se estima la probabilidad de aceptar contribuir con una aportación mensual según estrato, se limita únicamente a presentar el modelo logit estimado, en el entendido de que para su interpretación se procede de igual forma que se hizo en el subapartado 3.3.1. para el estrato doméstico bajo.
} 
A partir de los coeficientes de cada variable, mostrados en el cuadro de resultados siguiente, construimos el modelo logit correspondiente al estrato doméstico medio-alto y alto (DMA):

$$
Z_{\text {DMA }}=-1.481-0.693(\mathrm{G})-0.541(\mathrm{~A})+0.324(\mathrm{Y})+0.306(\mathrm{ICA})
$$

\subsubsection{Estrato doméstico general, Santa Ana Pacueco}

En esta localidad se entrevistó a 274 hogares equivalentes a $31.4 \%$ del total de la muestra. Debido a la no segmentación de esta submuestra el modelo logit calculado en este caso es válido para toda la población de Santa Ana Pacueco.

En este caso, las variables ingreso (Y), indice de cultura del agua (ICA), sexo $(\mathrm{G})$ y número de habitantes del hogar $(\mathrm{H})$, muestran el tipo de relación esperada con la variable dependiente. De manera que el modelo logit que permite obtener la probabilidad de aceptar el pago por parte de los hogares de Santa Ana Pacueco, es:

$$
\mathrm{Z}_{\mathrm{DSAP}}=-3.218+0.423(\mathrm{Y})+0.41(\mathrm{ICA})-0.242(\mathrm{G})-0.136(\mathrm{H})
$$

\subsection{DAP media según estrato}

Para el caso de la muestra, la DAP media equivale al promedio del monto que los hogares afirmaron estar dispuestos a contribuir mensualmente por mejoras en la calidad ambiental del meandro del Lerma. Sin embargo, para generalizar estos resultados al conjunto de la población hemos recurrido a un ejercicio de regresión lineal con la finalidad de encontrar el monto que los hogares de ambas localidades estarían dispuestos a pagar finalmente.

Con base a lo expresado por los hogares entrevistados en este estudio, se obtuvo que la DAP promedio de la muestra es de 50.43 pesos por mes en el caso de La Piedad de Cabadas (cuadro 7). Esta cantidad es menor en Santa Ana Pacueco, donde el mismo promedio asciende a 43.72 pesos por mes. ${ }^{14}$

$\mathrm{Si}$ bien estos promedios son representativos de toda la muestra, consideramos que los diferentes estratos de la población habrían de comportarse diferenciadamente en cuanto a su DAP, por lo que decidimos

\footnotetext{
${ }^{14}$ El cálculo de este promedio se realizó tomando en cuenta la máxima disposición a pagar expresada por los hogares de la muestra. Dentro del marco de la valoración contingente, dicha cantidad es obtenida al suponer cierta certidumbre acerca de la realización del proyecto ambiental. De esta manera procuramos estar lo más cerca posible del verdadero valor que tiene para un individuo el bien ambiental en cuestión, ya que se reducen las distorsiones que en la valoración pueden estar generando cuestiones como la desconfianza en las autoridades.
} 
segmentar a la población objetivo (hogares), siguiendo la clasificación de los usuarios hecha por el servicio local de agua potable.

\section{Cuadro 7}

DAP promedio de la muestra, según estrato

\begin{tabular}{lcc}
\hline \multicolumn{1}{c}{ Tipo de usuario } & Observaciones & DAP (media) \\
\hline Doméstico bajo LP & 103 & $\$ 38.47$ \\
Doméstico medio LP & 180 & $\$ 48.12$ \\
Doméstico medio-alto y alto LP & 173 & $\$ 59.97$ \\
Muestra La Piedad & 456 & $\$ 50.43$ \\
Santa Ana Pacueco (general) & 220 & $\$ 43.72$ \\
\hline
\end{tabular}

Fuente: elaboración propia.

En el mismo cuadro observamos cómo en la localidad michoacana, el estrato doméstico bajo y los hogares del doméstico medio tienen una DAP promedio menor que la de la muestra, mientras que en el último estrato, doméstico medio-ato y alto, la DAP es, en promedio, mayor a la media muestral. Estos comportamientos otorgan un punto a favor de la segmentación de los resultados por estrato pues permiten estimar con mayor precisión el monto total que los hogares de las localidades en cuestión estarían dispuestos a pagar por la mejor propuesta.

\subsection{Cálculo de la propuesta de pago mensual para la población}

Con la finalidad de encontrar el monto de la DAP que pueda generalizarse como pago propuesto para el conjunto de la población, así como las variables socioeconómicas que la determinan, hemos recurrido a la elaboración de un modelo de regresión basado en el método de mínimos cuadrados ordinarios (MCO).

Seguimos el mismo supuesto de comportamiento diferenciado de la muestra y hemos realizado el ejercicio de regresión para cada localidad y, en el caso de La Piedad, para cada estrato por separado con la finalidad de predecir de manera más adecuada la conducta de la población.

El cuadro 8 resume los resultados obtenidos en los ejercicios de regresión realizados para cada estrato. En este caso, la variable dependiente es la DAP expresada en términos monetarios por cada uno de los hogares entrevistados. Por otra parte, las variables que explican el comportamiento de la variable dependiente son, en general: nivel de ingreso mensual $(\mathrm{Y})$, número de habitantes del hogar $(\mathrm{H})$, nivel de escolaridad $(\mathrm{S})$, edad $(\mathrm{A})$, sexo $(\mathrm{G})$ del entrevistado, así como el grado en que la contaminación del río afecta a los integrantes del hogar (GA), según fueron especificadas con anterioridad (cuadro 2). 


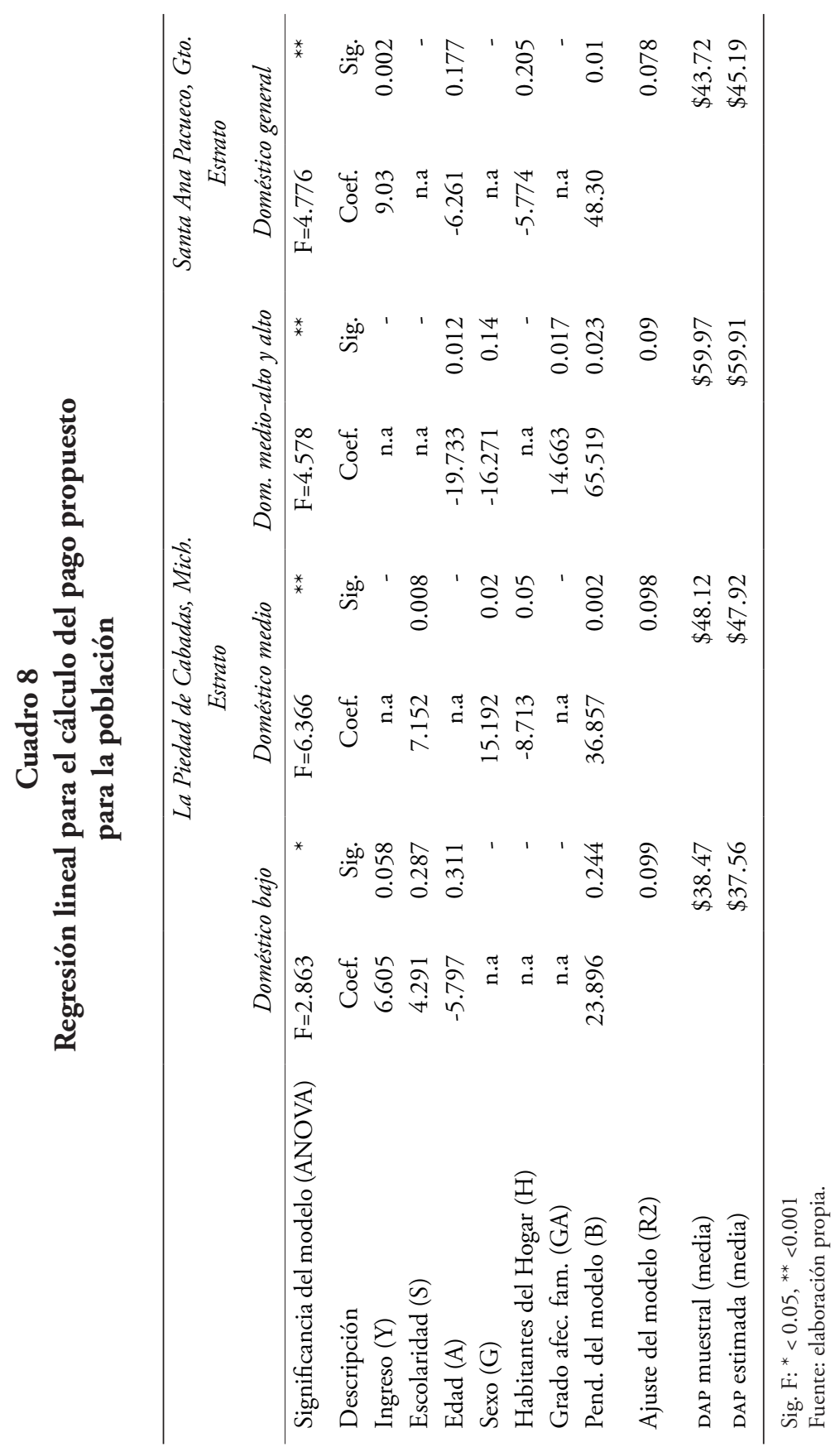


Los aspectos generales, en cuanto al significado de estos resultados, los conocemos ya debido a que no difieren mucho de los resultados de los modelos logísticos estimados para el cálculo de probabilidades que vimos antes. Como ejemplo, podemos ver para el caso del estrato doméstico bajo, el modelo que estima el monto que los hogares del mismo estarían dispuestos a pagar por revertir el estado de deterioro del meandro, es:

$$
\mathrm{DAP}_{\mathrm{DB}}=23.896+6.605(\mathrm{Y})+4.291(\mathrm{~S})-5.797(\mathrm{~A})
$$

La variable $\mathrm{DAP}_{\mathrm{DB}}$ o variable dependiente, es el monto que los hogares estarían dispuestos a pagar por la mejora ambiental que se propuso mediante el mercado hipotético que generamos, expresada en términos monetarios. Por tanto, los coeficientes de las variables explicativas, que se expresan en los mismos términos de la variable a la que explican, equivalen a la misma cantidad de pesos.

Así, si el coeficiente de la variable Y indica que, teóricamente, ante incrementos unitarios en la categoría de ingreso el monto de la DAP incrementará en 6.61 pesos, siempre que las demás variables del modelo permanezcan sin variaciones. A su vez, incrementos unitarios en la escolaridad aportarán 4.3 pesos al monto de la DAP, mientras que el incremento en una unidad de la variable edad restará 5.8 pesos a la DAP.

La constante del modelo, equivalente a 23.81 pesos, es el monto que cualquier hogar de la población correspondiente al estrato doméstico bajo estará dispuesto a pagar, independientemente de las demás variables. En otras palabras, la constante del modelo corresponde a la DAP autónoma. Hemos encontrado así, el monto del pago propuesto.

Este valor será aceptado, con cierto nivel de confianza, por el grueso de la población independientemente de los efectos generados por cualquier variable. La estimación de un modelo para cada estrato nos permite sugerir un pago distinto para cada uno de ellos a partir de sus propias características observadas (cuadro 9).

\section{Cuadro 9}

\section{Pago propuesto por localidad y estrato}

\begin{tabular}{clcc}
\hline Localidad & \multicolumn{1}{c}{ Estrato } & $\begin{array}{c}\text { Pago propuesto } \\
\text { (Coef. del Modelo) }\end{array}$ & Sig. $t$ \\
\hline La Piedad de Cabadas & Doméstico bajo & $\$ 23.896$ & -- \\
& Doméstico medio & $\$ 36.857$ & $*$ \\
& Dom. medio-alto y alto & $\$ 65.519$ & $*$ \\
Santa Ana Pacueco & Doméstico general & $\$ 48.303$ & $*$ \\
\hline
\end{tabular}

Sig. t: ${ }^{*}<0.05$

Fuente: elaboración propia. 
Estas cantidades pueden ser tomadas como referencia por las autoridades locales al momento de fijar el pago que cada hogar realizará mensualmente por concepto de mejoras ambientales al meandro del Lerma. Así, por ejemplo, es viable proponer un pago de 37 pesos mensuales para los hogares del estrato doméstico medio, ya que podemos afirmar, con un nivel de confianza mayor al 95\%, que ese monto será generalmente aceptado, siempre y cuando el hogar tenga la certeza de que su contribución será aplicada invariablemente al saneamiento ambiental del meandro, y que éste será eficaz.

\subsection{Agregación de los resultados: valor total de la DAP para la ZM}

Con el mvc el participante manifiesta sus preferencias tal como lo hace en el mercado un consumidor. El análisis de la información obtenida, permite inferir el excedente del consumidor, es decir, la diferencia entre lo que el consumidor pagará y lo que estaría dispuesto a pagar, determinando el beneficio esperado.

\subsubsection{Cálculo del valor total del bien ambiental}

Para calcular el valor total del bien ambiental tomaremos en cuenta la media de la DAP obtenida con la ayuda de los modelos estimados para cada estrato. En el cuadro 10 se resumen estos modelos, así como la media de la DAP calculada a partir de ellos.

\section{Cuadro 10 \\ Modelos de estimación de la DAP poblacional y media por estrato}

\begin{tabular}{|c|c|c|c|}
\hline Localidad & Estrato & $\begin{array}{l}\text { Modelo } \\
\text { estimado }\end{array}$ & $\begin{array}{l}\text { DAP Estimada } \\
\text { (media) }\end{array}$ \\
\hline \multirow{3}{*}{$\begin{array}{l}\text { La Piedad } \\
\text { de Cabadas }\end{array}$} & $\begin{array}{l}\text { Doméstico } \\
\text { bajo }\end{array}$ & $\begin{array}{l}\mathrm{DAP}_{\mathrm{DB}}=23.896+6.605(\mathrm{Y})+4.291(\mathrm{~S}) \\
-5.797(\mathrm{~A})\end{array}$ & $\$ 37.56$ \\
\hline & $\begin{array}{l}\text { Doméstico } \\
\text { medio }\end{array}$ & $\begin{array}{l}\mathrm{DAP}_{\mathrm{DM}}=36.857+15.192(\mathrm{G})+7.152(\mathrm{~S}) \\
-8.713(\mathrm{H})\end{array}$ & $\$ 47.92$ \\
\hline & $\begin{array}{l}\text { Dom. medio- } \\
\text { alto y alto }\end{array}$ & $\begin{array}{l}\mathrm{DAP}_{\mathrm{DMA}}=65.519+14.663(\mathrm{GA})- \\
16.271(\mathrm{G})-19.733(\mathrm{~A})\end{array}$ & $\$ 59.91$ \\
\hline $\begin{array}{l}\text { Santa Ana } \\
\text { Pacueco }\end{array}$ & $\begin{array}{l}\text { Doméstico } \\
\text { general }\end{array}$ & $\begin{array}{l}\mathrm{DAP}_{\mathrm{SAP}}=48.303+9.03(\mathrm{Y})-6.261(\mathrm{~A})- \\
5.774(\mathrm{H})\end{array}$ & $\$ 45.19$ \\
\hline
\end{tabular}

Fuente: elaboración propia. 
La DAP media estimada se extrapola al resto de la población como la cantidad promedio de los hogares de cada estrato y localidad. ${ }^{15}$ Así, se multiplica el total de hogares de cada estrato por su DAP media poblacional. La agregación de cada uno de estos subtotales indica la suma que se pagaría anualmente en la ZM para revertir el estado de deterioro ambiental del meandro del Lerma en ambas localidades (cuadro 11).

\section{Cuadro 11 \\ DAP promedio y total anual según tipo de usuario y localidad}

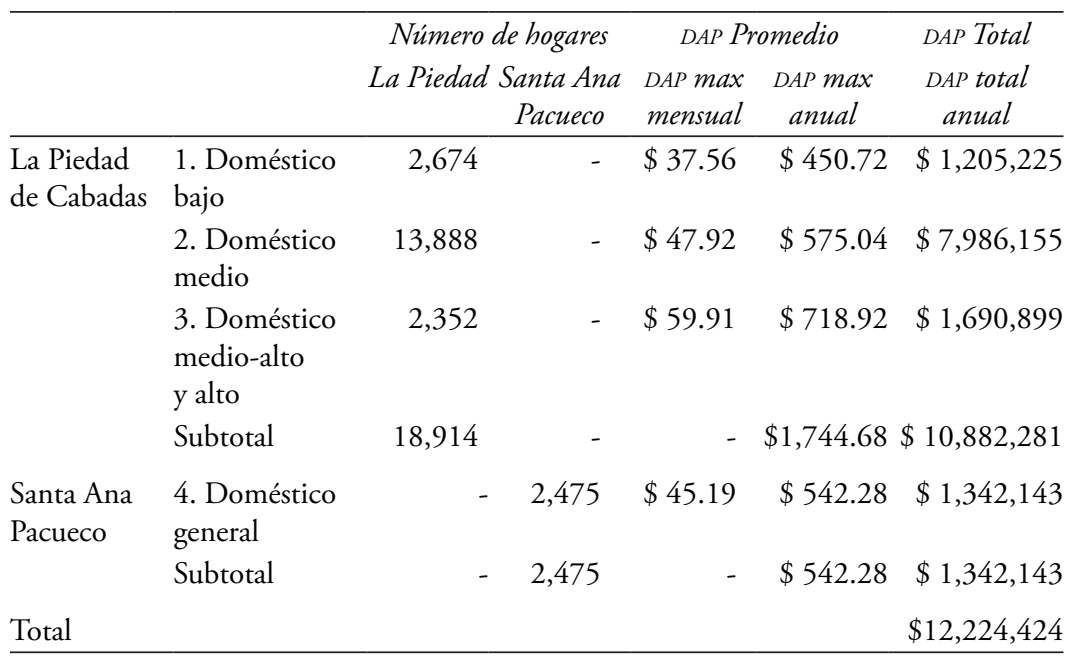

Fuente: elaboración propia.

De esta forma, la DAP agregada de los hogares de ambas localidades asciende a 12 millones 224 mil 424 pesos por ańo. Obsérvese que la participación de La Piedad es mucho mayor con relación a la de Santa Ana Pacueco, ya que la primera aportaría $89 \%$ de la DAP total anual. A su vez, el estrato de hogares de más peso por el monto total de su DAP, es el doméstico medio que aporta más de $73 \%$ de la Dap de La Piedad de Cabadas, y más de 66\% de la DAP total de ambas localidades; tal comportamiento se explica por el número de hogares pertenecientes a cada subgrupo de la población.

${ }^{15}$ Es importante señalar que la validez de esta extrapolación está determinada por el propio nivel de confianza y error máximo permisible alcanzado con el diseńo muestral descrito en el cuadro 1. Asimismo, en el marco contingente del método, el resultado de la extrapolación está también sujeto al cumplimiento del supuesto planteado a los entrevistados para expresar su máxima DAP, es decir, que "el total de su pago sería destinado para el propósito ambiental descrito (saneamiento del meandro) y que éste sería aplicado eficientemente”. 
Así, las mejoras ambientales propuestas para el meandro del Lerma son valuadas por el conjunto de la población en más de 12 millones de pesos anuales. La principal utilidad que tiene este cálculo es servir de base para la toma de decisiones que tiendan a revertir el deterioro ambiental del cauce natural del río Lerma en la zM. Por ejemplo, si confrontamos este valor frente a un proyecto de saneamiento y conservación del cauce natural del río Lerma en la zona metropolitana de La Piedad que costase, digamos, 150 millones con una vida útil de 20 ańos, significaría que el excedente social (beneficio social externo) de esa inversión sería de aproximadamente 90 millones de pesos, ${ }^{16}$ lo que justifica plenamente tal inversión.

\section{Conclusiones}

La problemática ambiental del meandro del Lerma constituye uno de los problemas más fuertes de la ZM La Piedad-Santa Ana Pacueco. En esta zona la contaminación del río es considerada el principal reto al que se enfrentan, sólo superado por la inseguridad, pero más grave que otros problemas socioeconómicos como el desempleo y la pobreza.

El estado de deterioro al que ha llegado el meandro del Lerma genera en los habitantes afecciones que van desde lo más ordinario, como la mala imagen, hasta las más preocupantes, como las enfermedades, deteriorando la calidad de vida de los habitantes de esta zona. Ante este escenario se confirma la necesidad de llevar a cabo proyectos que hagan posible la integración del meandro a la dinámica urbana de la ZM, empezando con acciones integrales de saneamiento que reviertan su deterioro ambiental.

Con este estudio se ha demostrado que existe la disposición por parte de los habitantes de la ZM para apoyar la realización de un proyecto que contribuya a este propósito. La población conoce la gravedad del problema ambiental pues sufre sus consecuencias, pero también acepta su responsabilidad como parte de la solución al deterioro del meandro. Esto se traduce en una amplia aceptación (DAP) para contribuir monetariamente a la solución. Con el MvC realizado se han podido conocer las características que determinan la cantidad de dinero que aportarían los hogares y la probabilidad de que se contribuya efectivamente al saneamiento del meandro.

Se ha encontrado que las variables que determinan la DAP, así como la probabilidad de que los hogares acepten realizar un pago por mejoras

\footnotetext{
${ }^{16}$ Esto es: $12,224,424$ pesos de DAP anual total de la ZM por 20 años es igual a $\$ 244,488,480$. Si a esta cantidad le restamos el total de la inversión en mejoras ambientales $(\$ 150,000,000)$, el resultado es $\$ 94,488,480$. Desde luego, a este cálculo habrá que ańadir los costos de operación, mantenimiento, financiamiento, depreciación, entre otros, dado que sólo es un caso hipotético.
} 
ambientales, difieren según estrato. Además del nivel de ingreso de los hogares, se encontró que aspectos como edad, escolaridad y sexo del encuestado, así como el número de habitantes y la cultura del agua del hogar, determinan la aceptación o rechazo a pagar por mejoras ambientales.

De acuerdo a la estratificación de hogares empleada para la población de La Piedad según tipo de usuario del servicio local de agua potable, el resultado en cuanto a la DAP fue consistente con el segmento socioeconómico, de forma que el estrato doméstico bajo obtuvo una DAP mensual de $\$ 38.47$, el estrato doméstico medio de $\$ 48.12$ y el medio alto y alto de $\$ 59.97$, con una media de $\$ 50.43$. Para el caso de la población de Santa Ana Pacueco, la DAP media estimada fue de 43.72 pesos mensuales.

Es importante señalar que estos resultados aportaron evidencia a favor de la hipótesis de que los estratos socioeconómicos en que se dividió a la población de la zM La Piedad-Santa Ana Pacueco, se comportan de forma independiente, con determinantes de la DAP y, por tanto, valoraciones diferentes a un nivel estadísticamente significativo.

Lo anterior también permite proponer la aplicación de un pago mensual diferenciado por parte de los hogares de la zona metropolitana, o bien, la aplicación de una cuota única a partir de la DAP autónoma del estrato doméstico bajo, que asegure su cumplimiento por todos los hogares de la zona, el cual debería ser de 23.89 pesos mensuales por hogar.

A partir de la DAP estimada por estratos y extrapolada al total de hogares con esas características en la ZM, ha sido posible estimar, además, cuál es el valor agregado que los hogares de la zona metropolitana conceden a las mejoras ambientales. En concreto, los proyectos, obras y actividades diversas requeridas para el saneamiento ambiental del meandro del río Lerma y su incorporación a la dinámica urbana de la zona metropolitana de La Piedad-Santa Ana, tiene un valor social de más de 12 millones de pesos anuales.

El cálculo económico del valor total por mejoras en la calidad ambiental del cauce natural del río, proporciona un elemento clave para conocer la viabilidad financiera del proyecto de saneamiento y restauración, pero también su rentabilidad social. Por su parte, la importancia de conocer los determinantes de la DAP radica en que ofrece la capacidad de predecir la reacción de los hogares ante el pago por mejoras ambientales a partir de la observación de algunas de sus características particulares.

Finalmente, encontrar evidencia del potencial apoyo ciudadano a un proyecto de saneamiento, basado en la participación de diferentes actores sociales, puede dar lugar a un proceso de sinergia que responda a la complejidad de la problemática existente. Sin embargo, se destaca la necesidad de acompańar los diversos proyectos ejecutivos con el desarrollo de una política ambiental integral que permita revertir el deterioro del entorno 
ecológico y contribuir a la formación de una mejor cultura ambiental, y de participación ciudadana con el objetivo de mejorar la calidad de vida de los habitantes de esta ZM.

\section{Agradecimientos}

Se agradece al Conacyt, así como al Consejo Estatal de Ciencia y Tecnología del Estado de Michoacán por el financiamiento del programa Fomix, proyecto no. 73881 "Saneamiento del cauce natural (meandro) del río Lerma e integración del mismo a la dinámica urbana de La Piedad, Michoacán”. Asimismo, se agradece a Nubia García Pérez, Miguel Reyna Rivera y Julio Morán por el apoyo con el trabajo de campo.

\section{Bibliografía}

Aguayo, Mariano (2007), "Cómo hacer una regresión logística con SPSS 'paso a paso'”, DocuWeb Fabis.org, Dot. núm. 0702012, Fundación Andaluza Beturia para la Investigación en Salud, pp. 1-16, $<$ http://www.fabis.org/html/archivos/docuweb/Regres_log_1r. pdf>, 28 de junio de 2011 .

Álvarez, Teresa, David Tafolla, Teresa Flores, Violeta Díaz, Diana Plancarte, Rocío Madrigal, Ruth Sánchez, Elvira Álvarez, Karina Hernández, Claudia Linares, Claudia García y Angélica Hernández (2011), "Salud del humano, animales domésticos y silvestres", reporte de investigación producto 13, en R. A. Rueda-Jasso (coord.), Informe Técnico Final del proyecto FOMIX-Conacyt 73881. Saneamiento del cauce natural (meandro) del rí Lerma e integración del mismo a la dinámica urbana de La Piedad, Michoacán, Universidad Michoacana de San Nicolás de Hidalgo, Morelia.

Arrow, Keneth, Robert Solow, Paul R. Portney, Edward E. Leamer, Roy Radner y Howard Schuman (1993), "Report of the NOAA panel on contingent valuation", Federal Register, 58 (10), The us National Archives and Records Administration, Washington, pp. 4601-4614.

Azqueta, Diego (1994), Valoración económica de la calidad ambiental, McGraw-Hill, Madrid. 
Azqueta, Diego, Mauricio Alvear, Lilia Domínguez y Raúl O’ Ryan (2007), Introducción a la economía ambiental, McGraw-Hill, Madrid.

Barton, David (2007), "Valoración contingente de las mejoras en la calidad del agua en Costa Rica”, en Rosi Ulate y Jesús Cisneros (comps.), Valoración económica, ecológica y ambiental. Análisis de casos en Iberoamérica, Editorial Universidad Nacional, Heredia, pp. 267-320.

Bernstein, Paula, Thomas C. Kinnaman y Mengqi Wu (2013), "Estimating willingness to pay for river amenities and safety measures associated with shale gas extraction", Eastern Economic Journal, 39 (1), Easton, pp. 28-44.

Egan, Kevin J., Joseph A. Herriges y John A. Downing (2009), "Valuing water quality as a function of water quality measures", American Journal of Agricultural Economics, 91 (1), Oxfor Journals, Oxford, pp. 106-123.

Franzen, Axel y Dominikus Vogl (2013), “Acquiescence and the willingness to pay for environmental protection: a comparison of the Issp, wvs and Evs", Social Science Quarterly, 94 (3), Southwest Social Science Association, pp. 637-659.

Gujarati, Damodar (2003), Econometría, McGraw-Hill, México.

Harrison, Glenn y Elisabeth Rutström (2008), "Experimental evidence on the existence of hypothetical bias in value elicitation methods", en Charles R. Plott y Vernon L. Smith (eds.), Handbook of experimental economics results, North Holland, Amsterdam, pp. 752-767.

Husted, Bryan W., Michael V. Russo, Carlos E. Basurto-Meza y Suzzanne G. Tilleman (2013), "An exploratory study of environmental attitudes and the willingness to pay for environmental certification in Mexico", Journal of Business Research, 67 (5), Elsevier, Amsterdam, pp. 892-899.

Ibarrarán, María Eugenia, Iván Islas y Eréndira Mayett (2003), "Valoración económica del impacto ambiental del manejo de residuos sólidos municipales: estudio de caso", Gaceta Ecológica, 67, 
Secretaría de Medio Ambiente y Recursos Naturales, México, pp. 69-82.

Ivehammar, Pernilla (2009), "The payment vehicle used in CV studies of environmental goods does matter", Journal of Agricultural and Resource Economics, 34 (3), Western Agricultural Economics Association, Milwaukee, pp. 450-463.

Labandeira, Xavier, Carmelo J. León y María José Vázquez (2007), Economía ambiental, Prentice Hall, Madrid.

López-Hernández, Martín, María Guadalupe Ramos-Espinosa y Javier Carranza-Fraser (2007), "Análisis multimétrico para evaluar la contaminación en el río Lerma y lago de Chapala", Hidrobiológica, vol. 17, UAM unidad Iztapalapa, México, pp. 17-30.

Mogas-Amorós, Joan (2004), "Métodos de preferencias reveladas y declaradas en la valoración de impactos ambientales", Ekonomiaz, 57, Gobierno Vasco, Victoria, pp. 12-29.

Navarro, Alejandro, Mauricio López y Georgina Caire (2004), "Estudio, análisis y propuestas para el fortalecimiento de los programas municipales de saneamiento ambiental existentes en la Cuenca Lerma Chapala”, documento de trabajo, Instituto Nacional de Ecología, México.

Ojeda, Monica Ilija, Alex S. Mayer y Barry D. Solomon (2008), "Economic valuation of environmental services sustained by water flows in the Yaqui River Delta”, Ecological Economics, 65 (1), Elsevier, New York, pp. 155-166.

Pucutay-Vásquez, Franck (2002), Los modelos logit y probit en la investigación social, Instituto Nacional de Estadística e Informática, Lima.

Riera, Pere, Dolores García, Bengt Kriström y Runar Brännlund (2005), Manual de economía ambiental y de los recursos naturales, Thomson, Madrid.

Rojas-Padilla, J., M. Pérez-Rincón y M. Peña-Varón (2001), “La valoración contingente: una alternativa para determinar la viabilidad financiera de proyectos de tratamiento de aguas residuales en 
zonas rurales de países tropicales", Cinara, Universidad del Valle, Cali, pp. 1-14.

Ruiz, Enrique (2009), "Lerma, sin solución", La Voz de Michoacán, 13 de abril, Morelia, pp. 4-5.

Sarmiento, Miguel Ángel y Antonio Prieto-Rodríguez (2005), “Métodos de valoración ambiental: un nuevo método basado en la variación del Producto Interno Bruto", Revista Catastro, 53, Dirección General del Catastro, Madrid, pp. 59-91.

Sotelo, Esthela, Nayeli Cardona, Alejandra Fregoso, Carlos Enríquez, Arturo Garrido, Georgina Caire y Helena Cotler (2005), Acciones estratégicas para la recuperación de la cuenca Lerma-Chapala: recomendaciones técnicas para las diecinueve subcuencas, Instituto Nacional de Ecología, México.

Soto-Montes de Oca, Gloria (2007), Agua: tarifas, escasezy sustentabilidad en las megaciudades: ¿Cuánto están dispuestos a pagar los habitantes de la ciudad de México?, Sistema de Aguas de la Ciudad de México-Universidad Iberoamericana-Centro de Estudios Jurídicos y Ambientales-Procuraduría Ambiental y del Ordenamiento Territorial del Distrito Federal, México.

Recibido: 23 de julio de 2011. Reenviado: 3 de octubre de 2011. Aceptado: 14 de mayo de 2013.

Dante Ariel Ayala-Ortiz. Mexicano. Cursó las licenciaturas en economía y biología en la Universidad Michoacana, es maestro en Integración Regional y Desarrollo Sustentable (Roskilde University, Dinamarca) y doctor en Economía Agroindustrial por la Universidad Autónoma Chapingo. Se desempeña como profesor investigador titular de la Universidad Michoacana de San Nicolás de Hidalgo, donde actualmente es jefe de la división de estudios de posgrado, Facultad de Economía. Es miembro del Sistema Nacional de Investigadores, nivel I, y coordinador del cuerpo académico en consolidación CA UMSNH 238 Estudios multidisciplinarios sobre Ambiente, Desarrollo y Sustentabilidad. Sus líneas de investigación vigentes son desarrollo rural sustentable, valoración económica de la calidad ambiental y, abasto y seguridad alimentaria. Entre sus publicaciones destacan: en coautoría, "La ecocondicionalidad como instrumento de 
política agrícola para el desarrollo rural sustentable en México", Gestión y Politica Pública, XVII (2), CIDE, México, pp. 315-353 (2008); en coautoría, "La multifuncionalidad de la agricultura campesina en la Meseta Purépecha: contribuciones metodológicas para su valoración”, Economía, Sociedad y Territorio, X (31), El Colegio Mexiquense, A.C., Zinacantepec, pp. 759-802 (2009); en coautoría, "Análisis comparativo de prácticas agrícolas sustentables en comunidades campesinas e indígenas de la Meseta Purépecha, México", Revista Iberoamericana de Economía Ecológica, vol. 13, Redibec, Barcelona, pp. 29-39 (2009).

Francisco Abarca-Guzmán. Mexicano. Economista por la Universidad Michoacana de San Nicolás de Hidalgo. Actualmente cursa la maestría en Administración y Política Pública en el Centro de Investigación y Docencia Económicas. Ha colaborado en diversos proyectos de investigación a cargo de la Universidad Michoacana, en la que también se ha desempeñado como profesor. Sus líneas de investigación, además de la economía ambiental, están relacionadas con el desarrollo social y la evaluación de políticas públicas. 